\title{
NFI-C Regulates Osteoblast Differentiation via Control of Osterix Expression
}

SEOUL

Joo-Cheol Park

Department of Oral Histology-Developmental Biology \& Dental Research Institute, School of Dentistry, Seoul National University, 101 Daehagro, Chongro-gu, Seoul, 110-749, Korea

\section{ABSTRACT}

In bone marrow, bone marrow stromal cells (BMSCs) have the capacity to differentiate into osteoblasts and adipocytes. Age-related osteoporosis is associated with a reciprocal decrease of osteogenesis and an increase of adipogenesis in bone marrow. In this study, we demonstrate that disruption of nuclear factor I-C (NFI-C) impairs osteoblast differentiation and bone formation, and increases bone marrow adipocytes. Interestingly, NFI-C controls postnatal bone formation but does not influence prenatal bone development. We also found decreased NFI-C expression in osteogenic cells from human osteoporotic patients. Notably, transplantation of Nfic-overexpressing BMSCs stimulates osteoblast differentiation and new bone formation, but inhibits adipocyte differentiation by suppressing PPAR $\gamma$ expression in $\mathrm{Nfic}^{-/-}$mice showing an age-related osteoporosis-like phenotype. Finally, NFI-C directly regulates Osterix expression but acts downstream of the BMP-2-Runx2 pathway. These results suggest that NFI-C acts as a transcriptional switch in cell fate determination between osteoblast and adipocyte differentiation in BMSCs. Therefore, regulation of NFI-C expression in BMSCs could be a novel therapeutic approach for treating age-related osteoporosis.

DISCLOSURE OF POTENTIAL CONFLICTS OF INTERESTS

The authors declare no conflicts of interest.

\section{NTRODUCTI ON}

Bone marrow stromal cells (BMSCs) have the capacity to differentiate into osteoblasts and adipocytes. Osteogenesis is regulated by severa growth and transcription factors, such as transforming growth factor $\beta$ (TGF- $\beta$ ), bone morphogenetic proteins (BMPs), Wnt, Hedgehog, Runx2 Osterix (Osx), and $\beta$-catenin, whereas adipogenesis is controlled by peroxisome proliferator-activated receptor gamma (PPAR $\gamma$ ). With aging, BMSCs become inclined to undergo differentiation into adipocytes, resulting in an increased number of adipocytes and a decreased number of osteoblasts in bone marrow. However, the mechanism underlying this differentiation switch remains unknown.

The nuclear factor I (NFI) family members of transcription factors are expressed from four highly conserved genes in mammals (named Nfia, $N f i b$, Nfic, and Nfix). All four NFI genes are expressed in human osteoblasts and osteoblast-like cell lines. In particular, Nfic mRNA is highly expressed in normal osteoblasts compared with other NFI family members. In addition, $\mathrm{Nfic}^{-1-}$ mice showed defects in alveolar bone formation in molar tooth sockets. However, the exact role of NFI-C in osteoblast differentiation and bone formation remains unclear. In the present study, we investigated the role of NFI-C in osteoblas differentiation and bone formation during osteogenesis.

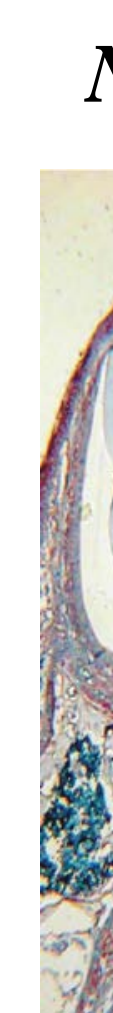

\section{$\sin 6$}

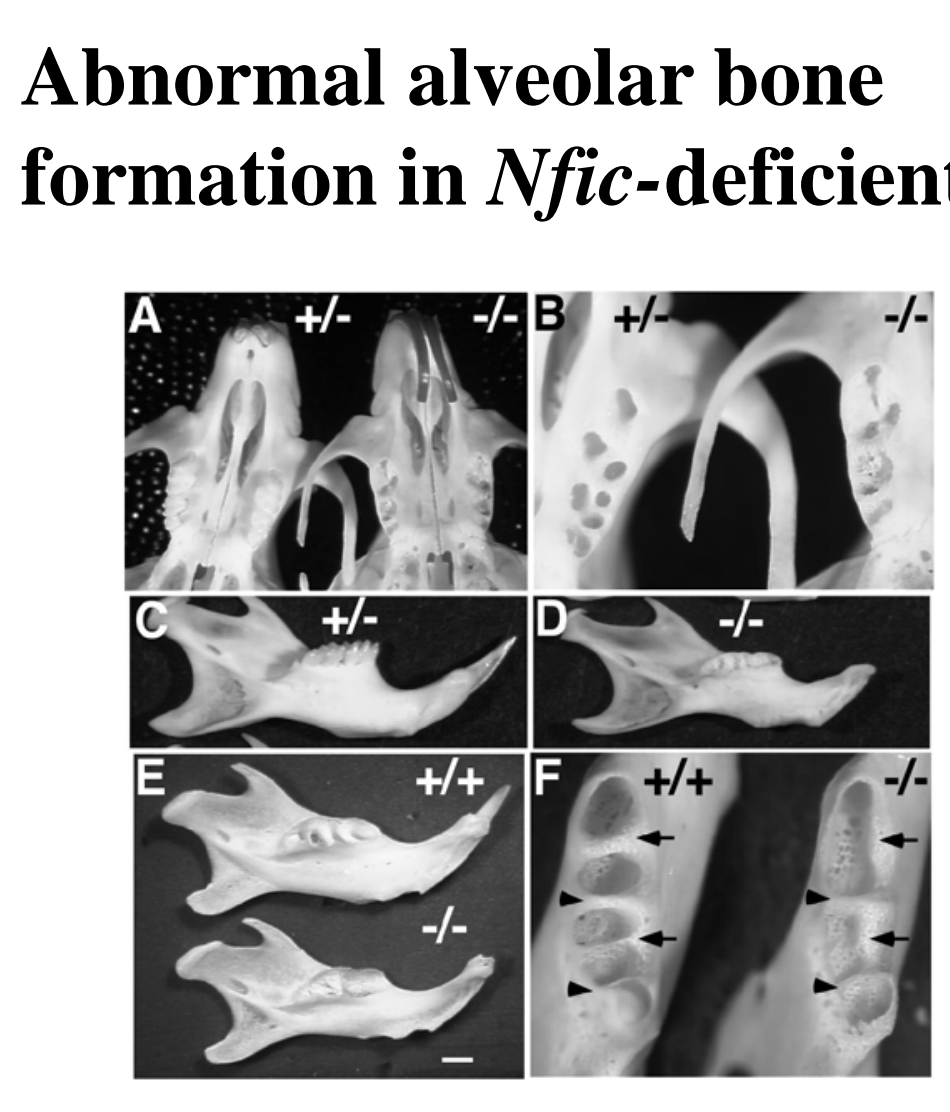

\section{METHODS}

Animals

- Nfic-mice were kindly provided by Dr. Richad

- Analyzed by micro-CT with a SkyScan scanner and the associated software.

Histology Analyse

a, TRAP, and IHC staining.

Cell Culture

BMSCs were isolated in tibia and femur of 6-week-old WT and $\mathrm{Nfic}^{-/}$mice Osteogenic differentiation - $\alpha$-MEM $+5 \%$ FBS, ascorbic acid (50 $\mu \mathrm{g} / \mathrm{ml}$ ), and -glycerophosphate $(10 \mathrm{mM})$.

Adipogenic differentiation - DMEM + 10\% FBS, insulin (10 $\mathrm{\mu g} / \mathrm{ml})$, examethasone $(1 \mu \mathrm{M})$, and 3-isobutyl-1-methylxan

Bone Marrow Cavity Transplantation of BMSCs

- Nfic-1-BMSCs were labeled with

- The cells were injected Nfic- or Osx-overexpressing BMSCs, or corresponding mock-infected (GFP-labeled) BMSCs $\left(1 \times 10^{6}\right.$ cells/femur in $10 \mu 1$ of $\alpha$-MFM $)$ into the bone marrow cavity of the left femur.

- Analyzed using micro-CT.

RT-PCR and Real-time PCR Analyses

Western Blot
ChIP Assays

Gene-Expression Profiling

- Publicly available gene expression datasets were downloaded from GEO

(accession number GSE35959). NFI-C mRNA expression (Probeset ID

213298_at) was analyzed between hBMSCs of osteoporotic patients

(hBMp.

Statistical Analyses

- All quantitative data are presented as the mean \pm S.D.

Statistical differences were analyzed by using

\section{RESULTS}
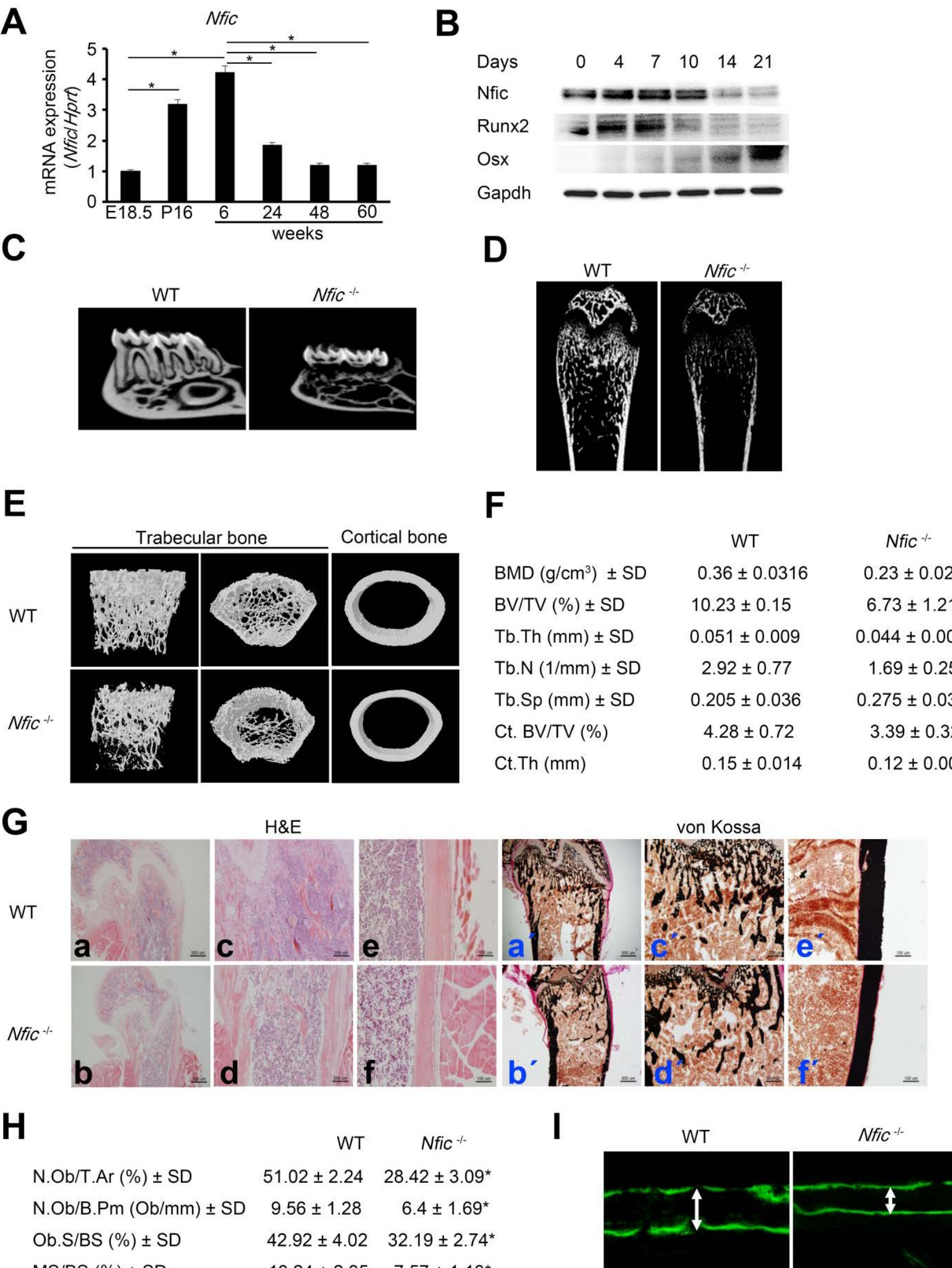

Figure 1. Nfic disruption impairs bone formation during postnatal osteogenesis. (A): Nfic expression was evaluated using real-time CR analyses in BMSCs derived from aged mice. (B): Western blot analyses. (C): Representative (B) mandible and (D): the (E): 3D micro-CT images. (F): and von Kossa staining (án $\left.\mathbf{a}^{\prime}-\mathrm{f}^{\prime}\right) . \quad(\mathbf{H})$ :
and $\begin{array}{ll}\text { Histomorphometric analyses. (I): Mineral } & \end{array}$ apposition rates (MAR).

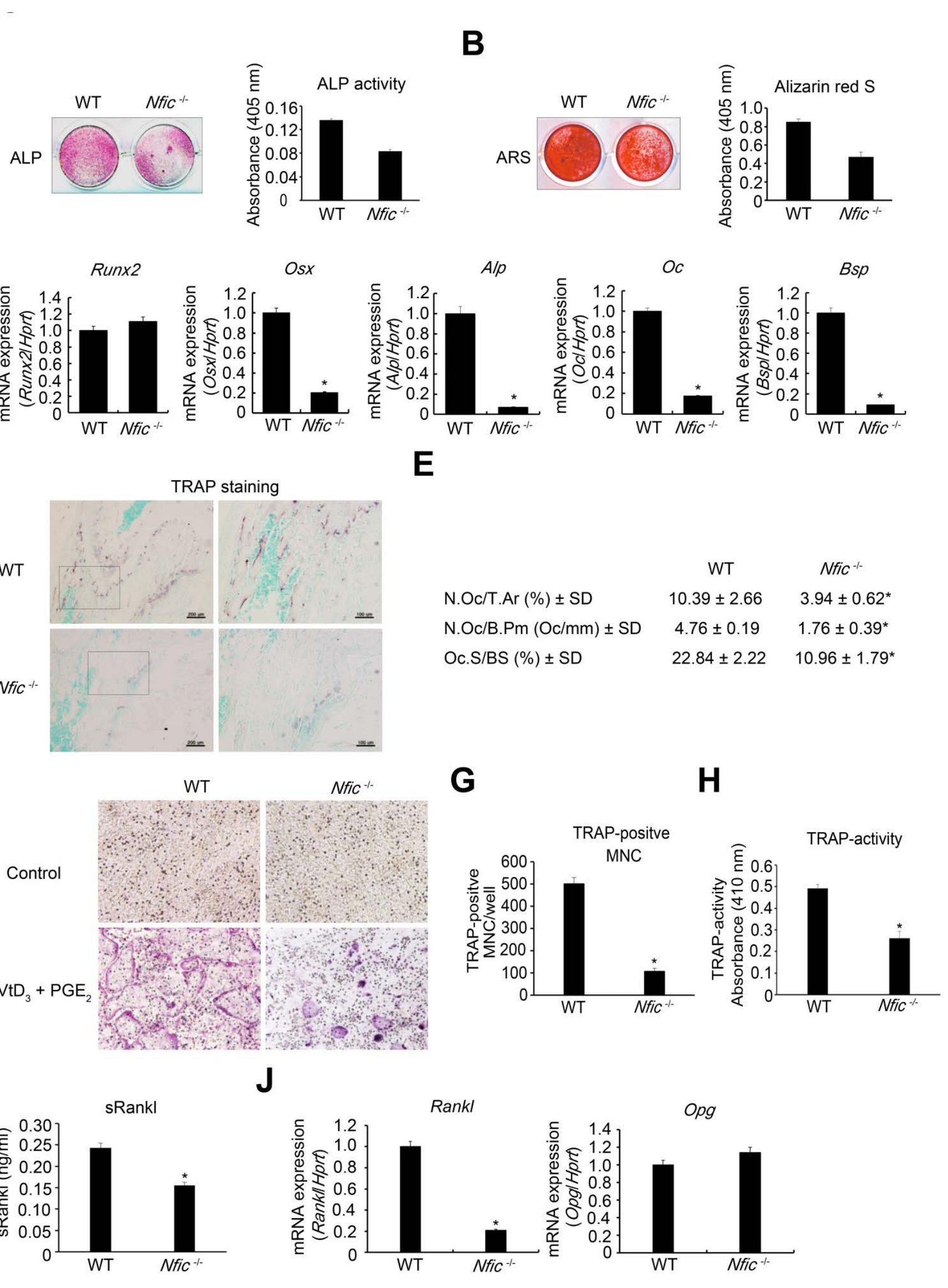

Figure 4. Nfic disruption impairs osteoblast differentiation and reduces osteoclast activity. (A): ALP staining and activity. (B): Alizarin red S staining (ARS). (C): Runx2, Osx, Alp, Oc, and Bsp expression. (D): TRAP staining of femurs from Histomorphometric analyses. (F): WT BMM were co-cultured with WT and $\mathrm{Nfic}^{-/}$primary $\mathrm{VitD}_{3}$ and $\mathrm{PGE}_{2}$, fixed, and stained for TRAP. (G): TRAP-positive multinucleated cells (MNCs) were counted in D. (H): TRAP activity was quantified in cell lysates. (I): sRankl levels were measured in
cell culture media using ELISA kits. (J): Rankl and Opg expression.

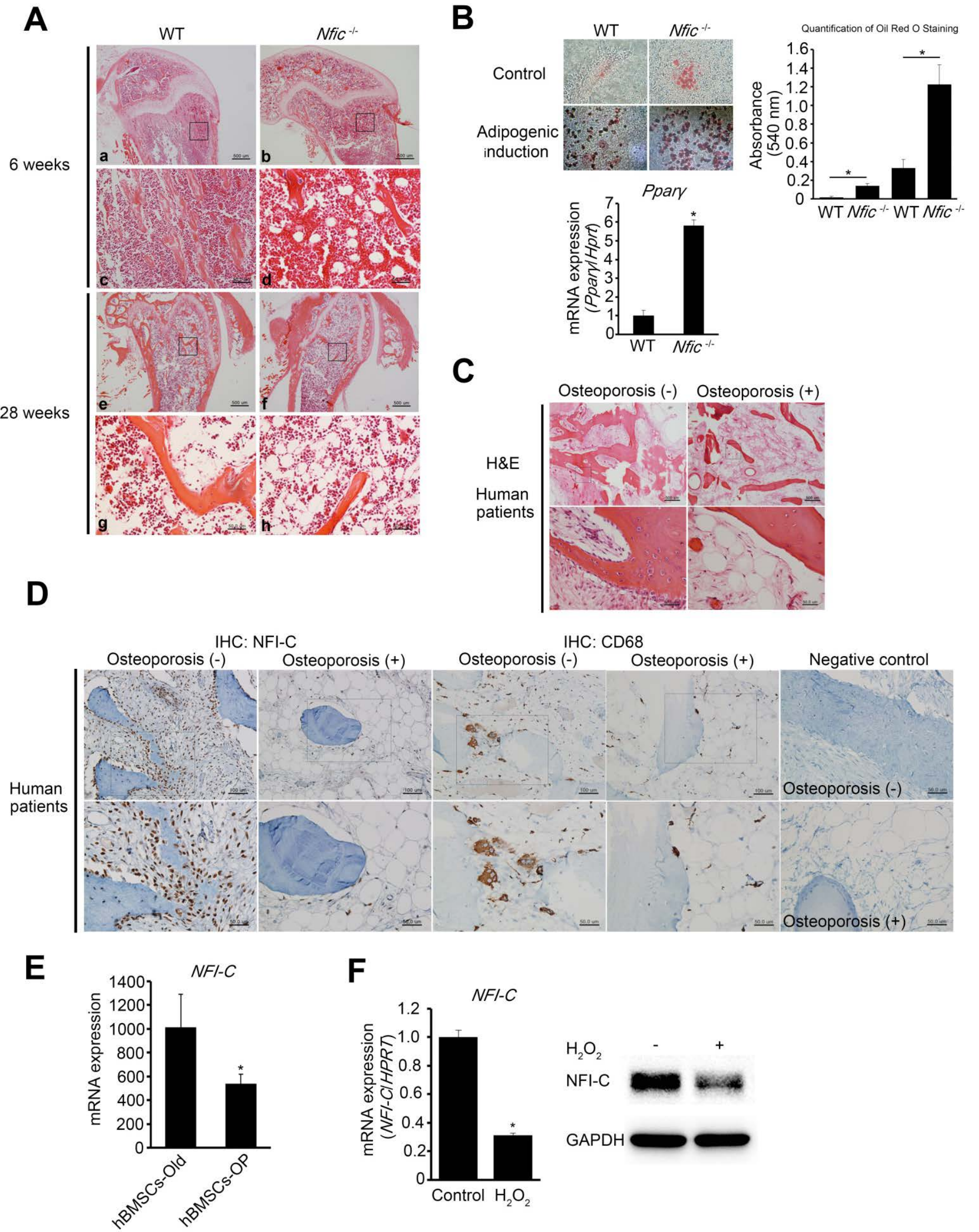

Figure 2. Nfic-deficiency increases bone marrow fat as seen in osteoporotic patients. (A): $\mathrm{H} \& \mathrm{E}$ staining. (B): Representative Oil Red $\mathrm{O}$ staining images (upper left panel) and quantification of oil red staining (upper right panel). (C): H\&E and (D): patient. (E): Expression of NFI-C mRNA was pexpression of NFI-C MRNA was deposited in gene expression dataset GSE35959 expression in hBMSCs.

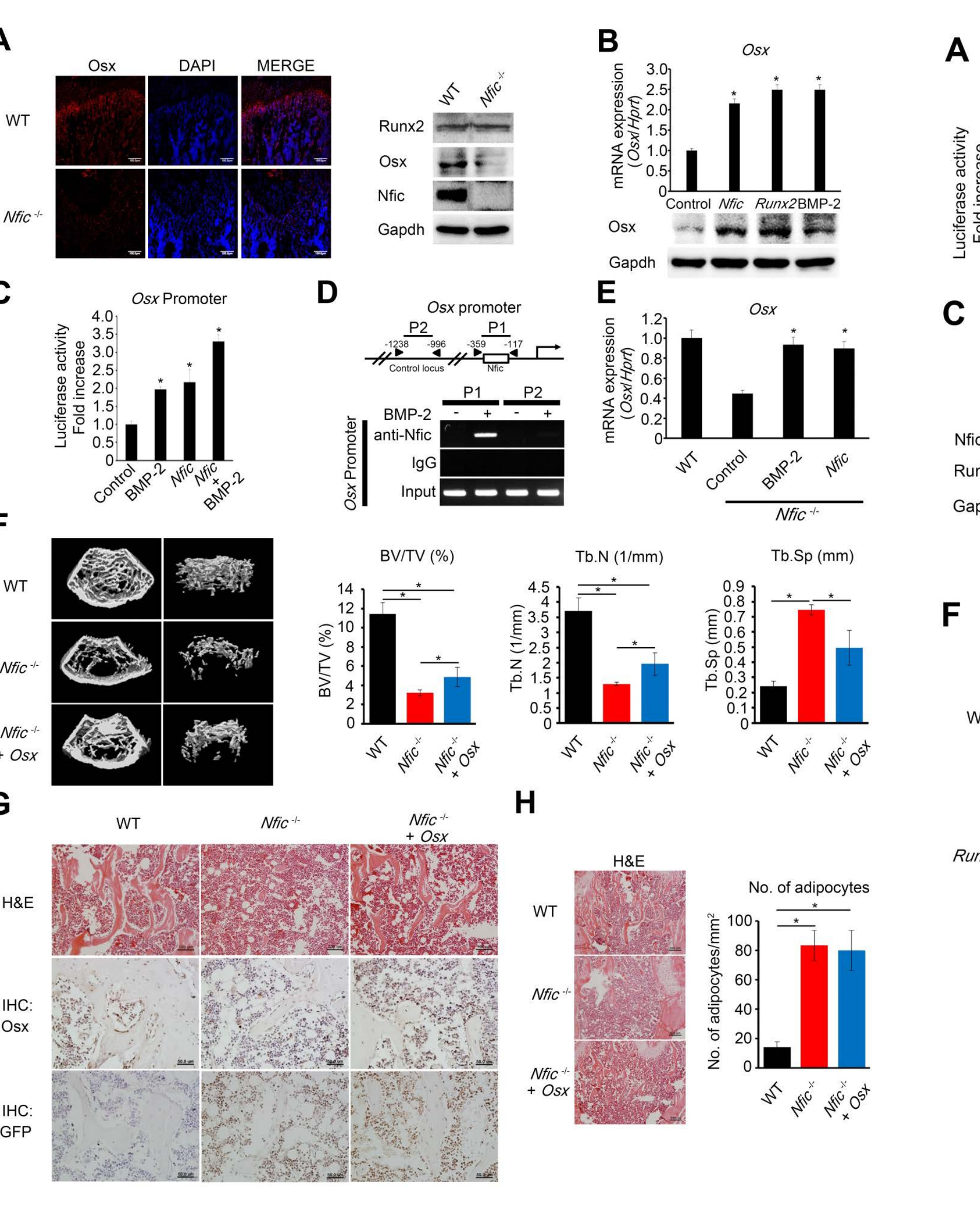

Figure 5. Nfic mediates BMP2-Runx2 induced Os $x$ expression. (A) Immunofluorescence staining of OsX (red). Western blot analyses. (B): Real-time PCR and western blot analyses. (C): Osx promoter activity. (D): ChIP analyses. P1 primers putative Nfic-binding motif. P2 primers:
negative control locus. (E): Nfic--o osteoblasts negative control locus. (E): Nfic/- osteoblasts
were treated with BMP-2 $(300 \mathrm{ng} / \mathrm{ml})$ and $/ \mathrm{or}$ were treated with BMP-2 $(300 \mathrm{ng} / \mathrm{ml})$ and $/ \mathrm{or}$
transfected with the Nfic expression vector. transfected with the Nfic expression vector.
Osx expression was analyzed using real-time Osx expression was analyzed using real-time
PCR. $n=3, *, P<.05$. (F): Representative micro-CT images and micro-CT quantification of the distal femurs in WT and $\mathrm{Nfic}^{-/-}$mice transplanted with Osx-overexpressing BMSC or mock-infected BMSCs at 10 weeks of age. (G): Histological analyses. (H): H\&E staining (left panel) and number of adipocytes.

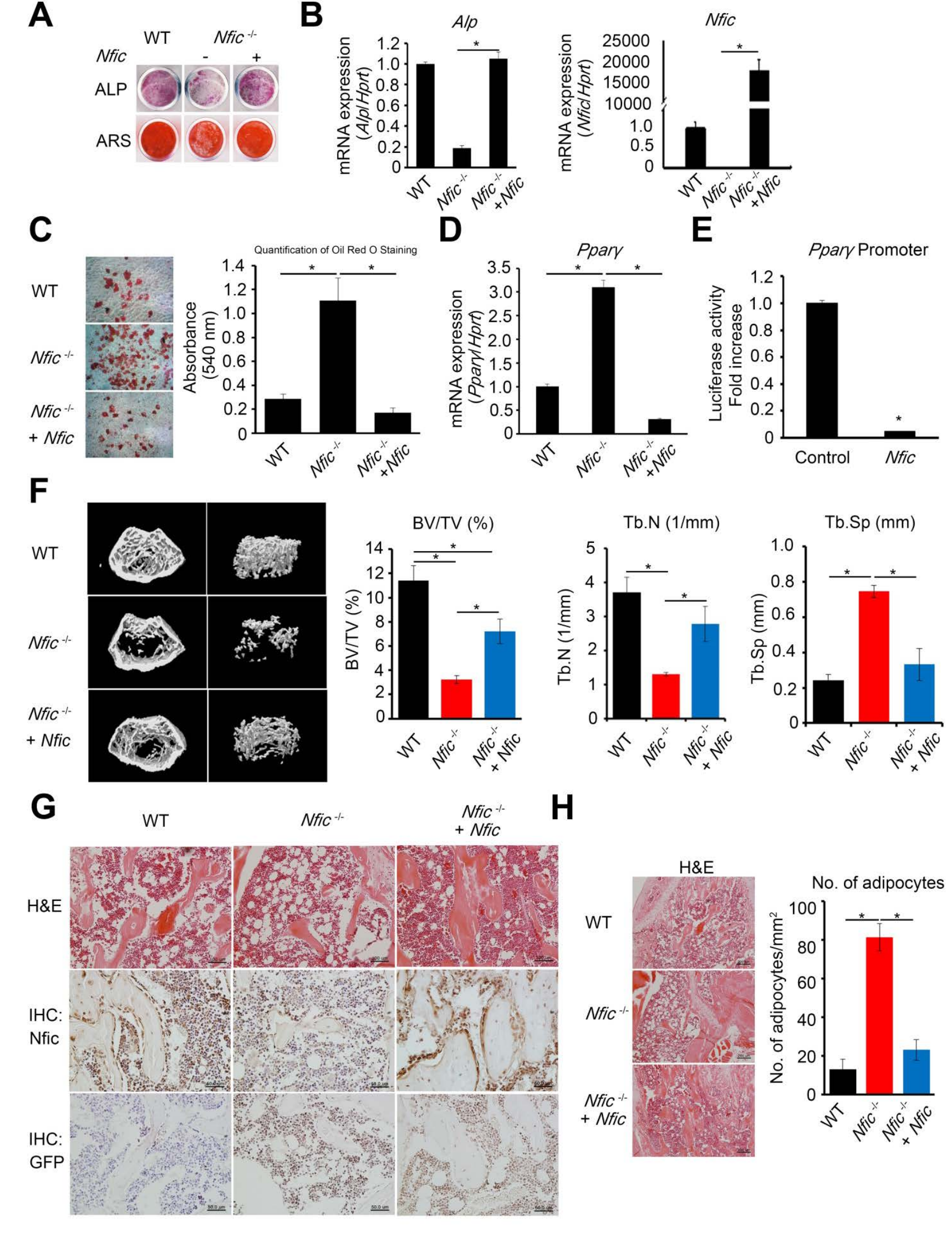

Figure 3. Nfic accelerates osteoblast differentiation and suppresses adipocyte differentiation. (A): ALP staining and Alizarin red $S$ staining. (B): Alp (left panel) and Nfic (right panel) expression. (C): Representative Oil Red O staining images (left panel) and quantification of oil red O staining (right panel). (D): Ppary expression. (E): Ppary promoter activity. (F): Representative micro-CT images and micro-CT quantification of transplanted with Nfic-overexpressing BMSCs or mock-infected BMSCs at 10 weeks of age. (G) Histological analyses. (H): H\&E staining (left panel) and number of adipocytes (right panel).
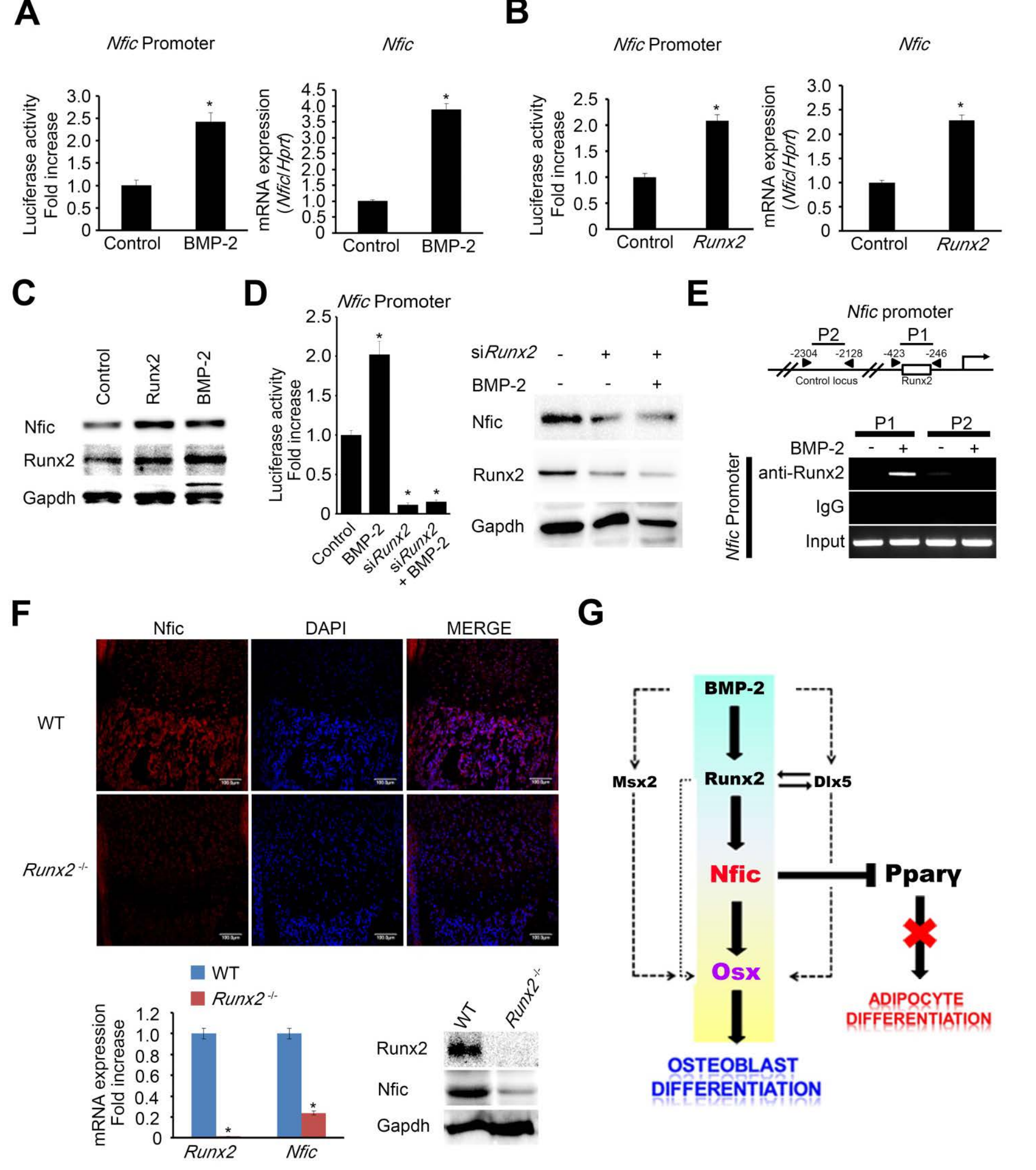

Figure 6. Runx2 mediates BMP-2-induced Nfic expression. (A and $\mathbf{B}$ ): Nfic promoter activity and mRNA expression. (C): Western blot analyses. (D): Nfic promoter activity. (E): ChIP analyses. P1 primers: putative Runx2-
binding motif. P2 primers: negative control binding motif. P2 primers: negative control
locus (F): Immunofluorescence staining of locus. (F): Immunofluorescence staining of
Nfic (Red) in femurs from E18.5 WT and Nfic (Red) in femurs from E18.5 WT and
Runx $2^{--}$mice. Total RNA and protein isolated Run $\times 2^{-/-}$mice. Total RNA and protein isolated
in calvarial bone from WT and Runx $2^{-/-}$mice. in calvarial bone from WT and Runx2-- mice. Runx $x$ and Nfic expression was assessed using
real-time PCR and western blot analyses. (G): real-time PCR and western blot analyses. (G):
A model of role of Nfic during osteoblast and adipocyte differentiation.

\section{SUMMARY \& CONCLUSI ON}

. We found an age-related decrease in Nfic expression in BMSCs.

$\mathrm{Nfic}^{-/}$mice show an age-related osteoporosis-like phenotype with decreased osteoblast differentiation and increased adipocyte differentiation.

Nfic overexpression reduced adipocyte differentiation through suppression of PPAR $\gamma$, but increased osteoblast differentiation in $\mathrm{Nic}^{-/-}$BMSC

4. Transplantation of Nfic-overexpressing BMSCs rescued an osteoporosis-like phenotype in $\mathrm{Nfic}{ }^{-/}$mice.

5. Nfic directly regulates Osx expression through the BMP-2 signaling pathway during osteoblast differentiation.

6. Runx2 acts upstream of Nfic and regulates Nfic expression through the BMP-2 signaling pathway.

These findings suggest that NFI-C is an important factor regulating the balance between osteoblast and adipocyte differentiation in BMSCs.

Taken together, our data suggest that NFI-C is a new candidate gene that causes osteoporosis. Therefore, regulation of NFI-C expression in BMSCs could be a novel therapeutic approach for treating osteoporosis. 